Article

\title{
Bäcklund Transformations for Integrable Geometric Curve Flows
}

\author{
Changzheng Qu ${ }^{1, *}$, Jingwei Han ${ }^{2}$ and Jing Kang ${ }^{3}$ \\ ${ }^{1}$ Department of Mathematics, Ningbo University, Ningbo 315211, China \\ ${ }^{2}$ School of Information Engineering, Hangzhou Dianzi University, Hangzhou 310018, China; \\ E-Mail: jingweih@hdu.edu.cn \\ ${ }^{3}$ Department of Mathematics, Northwest University, Xi'an 710069, China; \\ E-Mail: jingkang@nwu.edu.cn
}

* Author to whom correspondence should be addressed; E-Mail: quchangzheng@nbu.edu.cn; Tel.: +86-574-8760-9976.

Academic Editor: Roman M. Cherniha

Received: 1 May 2015 / Accepted: 30 July 2015 / Published: 3 August 2015

\begin{abstract}
We study the Bäcklund transformations of integrable geometric curve flows in certain geometries. These curve flows include the KdV and Camassa-Holm flows in the two-dimensional centro-equiaffine geometry, the $\mathrm{mKdV}$ and modified Camassa-Holm flows in the two-dimensional Euclidean geometry, the Schrödinger and extended Harry-Dym flows in the three-dimensional Euclidean geometry and the Sawada-Kotera flow in the affine geometry, etc. Using the fact that two different curves in a given geometry are governed by the same integrable equation, we obtain Bäcklund transformations relating to these two integrable geometric flows. Some special solutions of the integrable systems are used to obtain the explicit Bäcklund transformations.
\end{abstract}

Keywords: invariant geometric flow; Bäcklund transformation; integrable system; differential invariant

MSC classifications: $37 \mathrm{~K} 35,37 \mathrm{~K} 25$, 53A55 


\section{Introduction}

Bäcklund transformations are a powerful tool to explore various properties of integrable nonlinear partial differential equations $[1,2]$. They can be used to obtain more exact solutions of integrable systems from a particular solution. The classical Bäcklund transformations are local geometric transformations, which are used to construct surfaces of constant negative Gaussian curvature [1]. This provides a geometric construction of new pseudospherical surfaces from a particular solution of an integrable partial differential equation. Indeed, solutions of the sine-Gordon equation describe pseudospherical surfaces. Applying Bäcklund transformations $n$ times to a particular solution of sine-Gordon equation, one can obtain a family of solutions of sine-Gordon equation. These solutions can be obtained using the Bianchi's permutability formula through purely algebraic means [2]. In [3], Chern and Tenenblat performed a complete classification to a class of nonlinear evolution equations which describe pseudospherical surfaces. It is noted that a nonlinear PDE describes pseudospherical surface if it admits $s l(2)$ prolongation structure. More generally, a Bäcklund transformation is typically a system of first-order partial differential equations relating two equations, and usually depending on an additional parameter. In particular, a Bäcklund transformation which relates solutions of the same equations is called an auto-Bäcklund transformation. In [4], Wahlquist and Estabrook [4] provides a systematic method to construct Bäcklund transformations of integrable systems by using the prolongation structure approach. Other effective methods to construct Bäcaklund transformations of integrable systems were also proposed in a number of literatures, see for example [2,5-12] and many more references.

A particular nice feature of integrable systems is their relationship with invariant geometric flows of curves and surfaces in certain geometries. Those flows are invariant with respect to the symmetry groups of the geometries [13]. A number of integrable equations have been shown to be related to motions of curves in Euclidean geometry, centro-equiaffine geometry, affine geometry, homogeneous manifolds and other geometries etc., and many interesting results have been obtained [14-41]. Such relationship is helpful to explore geometric realization of several properties of integrable systems, for example, bi-Hamiltonian structure, recursion operator, Miura transformation and Bäcklund transformation etc. On the other hand, the topological properties of closed curves are shown to be related to the infinite number of symmetries and the associated sequence of invariants [11]. The relationship between integrable systems and geometric curve flows in $\mathbb{R}^{3}$ was studied in 1970s by Hasimoto [14], who showed that the integrable cubic Schrödinger equation is equivalent to the binormal motion flow of space curves in $\mathbb{R}^{3}$ (called vortex-filament flow or localized induction equation) by using a transformation relating the wave function of the Schrödinger equation to the curvature and torsion of curves ( so-called Hasimoto transformation). Furthermore, using the Hasimoto transformation, Lamb [16] verified that the mKdV equation and the sine-Gordon equations arise from the invariant curve flows in $\mathbb{R}^{3}$. Marí-Beffa, Sanders and Wang [25] noticed that the Hasimoto transformation is a gauge transformation relating the Frenet frame and parallel frame. The well-known integrable equations including the $\mathrm{KdV}$ equation, the modified $\mathrm{KdV}$ equation, the Sawada-Kotera equation,the Kaup-Kuperschmidt equation and Boussinesq equation were also shown to arise from the invariant plane or space curve flows respectively in centro-equiaffine geometry [18,21,35,40], Euclidean geometry [15,17,21], two-dimensional affine geometry [21,40], projective geometry $[37,39]$ and three-dimensional affine geometry [23]. 
In this paper, we are mainly concerned with Bäcklund transformations for integrable geometric curve flows in certain geometries. Our work is inspired by the following result.

Proposition 1.1. [26] Let $\gamma(s)$ be a smooth curve of constant torsion $\tau$ in $\mathbb{R}^{3}$, parametrized by arclength s. Let $\mathrm{T}, \mathrm{N}$ and $\mathbf{B}$ be a Frenet frame, and $k(s)$ the curvature of $\gamma$. For any constant $C$, suppose $\beta=\beta(s, k(s), C)$ is a solution of the differential equation

$$
\frac{d \beta}{d s}=C \sin \beta-k
$$

then

$$
\tilde{\gamma}(s)=\gamma(s)+\frac{2 C}{C^{2}+\tau^{2}}(\cos \beta \mathbf{T}+\sin \beta \mathbf{N})
$$

is a curve of constant torsion $\tau$, also parametrized by arclength $s$.

Note that this transformation can be obtained by restricting the classical Bäcklund transformation for pseudospherical surfaces to the asymptotic lines of the surfaces with constant torsion.

We will restrict our attention to the geometric plane curve flows

$$
\gamma_{t}=f \mathbf{N}+g \mathbf{T}
$$

and space curve flows

$$
\gamma_{t}=f \mathbf{T}+g \mathbf{N}+h \mathbf{B}
$$

in Euclidean, centro-equiaffine and affine geometries, where $\mathbf{T}$ and $\mathbf{N}$ in Equation (2) denote frame vectors of planar curves, and $\mathbf{T}, \mathbf{N}$ and $\mathbf{B}$ in Equation (3) are frame vectors of spacial curves, $f, g$ and $h$ depend on the curvatures of the curves $\gamma$ and their derivatives with respect to the arclength parameter, namely, these geometric flows are invariant with respect to the symmetry groups of the geometries.

For a planar or a spacial curve $\gamma(t, s)$ in a given geometry, let $\tilde{\gamma}(t, s)$ be another curve related to $\gamma$ through the following Bäcklund transformation

$$
\tilde{\gamma}(t, s)=\gamma(t, s)+\alpha(t, s) \mathbf{N}+\beta(t, s) \mathbf{T}
$$

or

$$
\tilde{\gamma}(t, s)=\gamma+\alpha(t, s) \mathbf{T}+\beta(t, s) \mathbf{N}+\chi(t, s) \mathbf{B} .
$$

Throughout the paper, we assume that both curve flows for $\gamma$ and $\tilde{\gamma}$ are governed by the same integrable system, that means the curvatures of the curves $\tilde{\gamma}$ determined by the flows (4) or (5) satisfy the integrable systems as for the curves $\gamma$. It turns out that the functions $\alpha(t, s), \beta(t, s)$ and $\chi(t, s)$ for space case and $\alpha(t, s)$ and $\beta(t, s)$ for planar case satisfy systems of nonlinear evolution equations. Solving these systems then yields Bäcklund transformations between the two flows for $\gamma$ and $\tilde{\gamma}$.

The outline of this paper is as follows. In Section 2, we first study the Bäcklund transformations of planar curve flows in $\mathbb{R}^{2}$, which include the modified $\mathrm{KdV}$ flow and the modified Camassa-Holm flow. Bäcklund transformations of integrable space curve flows in $\mathbb{R}^{3}$ including the Schrödinger flow and the extended Harry-Dym flow will be discussed in Section 3. In Section 4, we consider the Bäcklund transformations of the $\mathrm{KdV}$ and Camassa-Holm flows for planar curves in centro-equiaffine geometry. Finally in Section 5, we discuss the Bäcklund transformations of the Sawada-Kotera flow in two-dimensional affine geometry. 


\section{Bäcklund Transformations of Integrable Curve Flows in $\mathbb{R}^{2}$}

The invariant geometric curve flows in $\mathbb{R}^{2}$ were discussed extensively from many points of view in the last three decades. A number of interesting results have been obtained. It was shown that the non-stretching plane curve flows in $\mathbb{R}^{2}$ are related closely to the integrable systems including the modified KdV equation [15,17,21] and the modified Camassa-Holm equation [42]. In this section, we consider the Bäcklund transformations of those integrable flows.

Let us consider the flows for planar curves in $\mathbb{R}^{2}$, governed by

$$
\gamma_{t}=f \mathbf{n}+h \mathbf{t}
$$

where $\mathbf{t}$ and $\mathbf{n}$ denote the unit tangent and normal vectors of the curves, respectively, which satisfy the Serret-Frenet formulae

$$
\mathbf{t}_{s}=k \mathbf{n}, \quad \mathbf{n}_{s}=-k \mathbf{t}
$$

where $k$ is the curvature of the curve $\gamma, s$ is the arclength of the curve and $d s=g d p, p$ is a free parameter. The velocities $f$ and $h$ in Equation (6) depend on $k$ and it's derivatives with respect to the arclength parameter $s$. Let $\theta$ be the angle between the tangent and a fixed direction. Then $\mathbf{t}=(\cos \theta, \sin \theta)$, $\mathbf{n}=(-\sin \theta, \cos \theta)$, and $d \theta=k d s$. Based on the flow (6), it is easy to show that the time evolutions of those geometric invariants are given by [17]

$$
\begin{aligned}
\mathbf{t}_{t} & =\left(f_{s}+k h\right) \mathbf{n}, \\
\mathbf{n}_{t} & =-\left(f_{s}+k h\right) \mathbf{t}, \\
g_{t} & =g\left(h_{s}-k f\right),
\end{aligned}
$$

and

$$
\begin{aligned}
\theta_{t} & =\left(f_{s}+k h\right), \\
k_{t} & =\left(\frac{d \theta}{d s}\right)_{t}=f_{s s}+k_{s} h+k^{2} f .
\end{aligned}
$$

Assume that the flow is intrinsic, namely the arclength does not depend on time. Then equation

$$
h_{s}=k f
$$

follows from Equation (8).

\subsection{The Modified KdV Flow in $\mathbb{R}^{2}$}

In [17], Goldstein and Petrich proved that the modified KdV equation arises from an non-stretching curve flow in Equation $\mathbb{R}^{2}$. Indeed, let $f=k_{s}, h=\frac{1}{2} k^{2}$ in Equation (6), then $k$ satisfies the modified $\mathrm{KdV}$ equation

$$
k_{t}=k_{s s s}+\frac{3}{2} k^{2} k_{s}
$$

The corresponding curve flow is

$$
\gamma_{t}=k_{s} \mathbf{n}+\frac{1}{2} k^{2} \mathbf{t}
$$


which is the so-called modified KdV flow [17].

Let $\tilde{\gamma}$ be another curve in $\mathbb{R}^{2}$ related to $\gamma$ by

$$
\tilde{\gamma}(t, s)=\gamma(t, s)+\alpha(t, s) \mathbf{n}+\beta(t, s) \mathbf{t} .
$$

Assume that $\tilde{\gamma}(t, s)$ is also governed by the modified $\mathrm{KdV}$ flow, namely it satisfies

$$
\tilde{\gamma}_{t}=\tilde{k}_{\tilde{s}} \tilde{\mathbf{n}}+\frac{1}{2} \tilde{k}^{2} \tilde{\mathbf{t}}
$$

where $\tilde{s}$ is the arclength parameter of $\tilde{\gamma} ; \tilde{\mathbf{t}}$ and $\tilde{\mathbf{n}}$ denote the unit tangent and normal vector of $\tilde{\gamma}$, respectively. A direct computation shows that $\tilde{\mathbf{t}}$ and $\tilde{\mathbf{n}}$ are related to $\mathbf{t}$ and $\mathbf{n}$ by

$$
\tilde{\mathbf{t}}=\frac{F_{0} \mathbf{t}+G_{0} \mathbf{n}}{\sqrt{F_{0}^{2}+G_{0}^{2}}}, \quad \tilde{\mathbf{n}}=-\frac{G_{0} \mathbf{t}+F_{0} \mathbf{n}}{\sqrt{F_{0}^{2}+G_{0}^{2}}},
$$

where

$$
F_{0}=1+\beta_{s}-k \alpha, \quad G_{0}=\alpha_{s}+k \beta .
$$

It is inferred from Equation (13) that

$$
\tilde{\gamma}_{t}=\left[\alpha_{t}+k_{s}+\left(k_{s s}+\frac{1}{2} k^{3}\right) \beta\right] \mathbf{n}+\left[\beta_{t}+\frac{1}{2} k^{2}-\left(k_{s s}+\frac{1}{2} k^{3}\right) \alpha\right] \mathbf{t} .
$$

Differentiating Equation (13) with respect to $s$, after using the Serret-Frenet formulae (7), yields

$$
\tilde{\gamma}_{s}=\left(\left(1+\beta_{s}-k \alpha\right) \mathbf{t}+\alpha_{s}+k \beta\right) \mathbf{n}:=F_{0} \mathbf{t}+G_{0} \mathbf{n} .
$$

It follows that the arclength $\tilde{s}$ of $\tilde{\gamma}$ is related to $s$ of $\gamma$ by

$$
d \tilde{s}=\sqrt{F_{0}^{2}+G_{0}^{2}} d s .
$$

Furthermore, differentiating Equation (18) with respect to $\tilde{s}$, and using Equation (19) yields

$$
\tilde{k} \tilde{\mathbf{n}}=\tilde{\gamma}_{\tilde{s} \tilde{s}}=\left(F_{0}^{2}+G_{0}^{2}\right)^{-2}\left(F_{1} \mathbf{t}+G_{1} \mathbf{n}\right):=F_{2} \mathbf{t}+G_{2} \mathbf{n},
$$

where

and

$$
F_{2}=\frac{F_{1}}{\left(F_{0}^{2}+G_{0}^{2}\right)^{2}}, \quad G_{2}=\frac{G_{1}}{\left(F_{0}^{2}+G_{0}^{2}\right)^{2}}
$$

$$
\begin{aligned}
F_{1}= & -k\left(1+\beta_{s}-k \alpha\right)^{2}\left(\alpha_{s}+k \beta\right)+\left(\beta_{s s}-k_{s} \alpha-2 k \alpha_{s}-k^{2} \beta\right)\left(\alpha_{s}+k \beta\right)^{2} \\
& -\left(1+\beta_{s}-k \alpha\right)\left(\alpha_{s}+k \beta\right)\left(\alpha_{s s}+k_{s} \alpha+k \alpha_{s}\right), \\
G_{1}= & k\left(1+\beta_{s}-k \alpha\right)^{3}+k\left(1+\beta_{s}-k \alpha\right)\left(\alpha_{s}+k \beta\right)^{2}\left(\alpha_{s s} k_{s} \alpha+k \alpha_{s}\right) \\
& -\left(1+\beta_{s}-k \alpha\right)\left(\alpha_{s}+k \beta\right)\left(\beta_{s s}-k_{s} \alpha-k \alpha_{s}\right) .
\end{aligned}
$$

From Equation (20), we also have

$$
\begin{aligned}
& \tilde{k}_{\tilde{s}}=\frac{F_{0}\left(G_{2 s}+k F_{2}\right)-G_{0}\left(F_{2 s}-k G_{2}\right)}{F_{0}^{2}+G_{0}^{2}}:=F_{3}, \\
& \tilde{k}^{2}=-\frac{G_{0}\left(G_{2 s}+k F_{2}\right)+F_{0}\left(F_{2 s}-k G_{2}\right)}{F_{0}^{2}+G_{0}^{2}}:=G_{3} .
\end{aligned}
$$


Substituting Equations (15), (17) and (21) into Equation (14), we see that the modified KdV flow is invariant with respect to the Bäcklund transformation (13) if and only if $\alpha$ and $\beta$ satisfy the following system

$$
\begin{aligned}
& \alpha_{t}+k_{s}+\beta\left(k_{s s}+\frac{1}{2} k^{3}\right)=\frac{1}{\sqrt{F_{0}^{2}+G_{0}^{2}}}\left(\frac{1}{2} G_{0} G_{3}+F_{0} F_{3}\right), \\
& \beta_{t}+\frac{1}{2} k^{3}-\alpha\left(k_{s s}+\frac{1}{2} k^{3}\right)=\frac{1}{\sqrt{F_{0}^{2}+G_{0}^{2}}}\left(\frac{1}{2} F_{0} G_{3}-G_{0} F_{3}\right) .
\end{aligned}
$$

Theorem 2.1. The modified KdV flow (12) is invariant with respect to the Bäcklund transformation (13) if $\alpha(t, s)$ and $\beta(t, s)$ satisfy the system (22), where $G_{0}, F_{0}, F_{3}$ and $G_{3}$ are given in Equations (16) and (21).

It is noticed that a class of Bäcklund transformations for smooth and discrete plane curves in Euclidean space governed by the modified $\mathrm{KdV}$ equation were discussed in [12], which are derived by using the Bäcklund transformations of the potential modified KdV equation.

\subsection{The Modified Camassa-Holm Flow}

The modified Camassa-Holm equation

$$
m_{t}+\left(\left(u^{2}-u_{s}^{2}\right) m\right)_{s}+a u_{s s s}=0, \quad m=u-u_{s s}
$$

can be derived using the general approach of the tri-Hamiltonian duality from the modified KdV equation [42]. A direct consequence of such approach shows us that the modified Camassa-Holm equation is an integrable equation with bi-Hamiltonian structure. Interestingly, it has peaked solutions and can describe wave breaking phenomena [43]. It was also shown in [43] that the modified Camassa-Holm equation arises from a non-stretching planar curve flow in $\mathbb{R}^{2}$. Indeed, let $f=u_{s}$, $h=\frac{1}{2}\left(u^{2}-u_{s}^{2}\right)$ in Equation (6), then the corresponding modified Camassa-Holm flow is

$$
\gamma_{t}=u_{s} \mathbf{n}+\frac{1}{2}\left(u^{2}-u_{s}^{2}\right) \mathbf{t}
$$

where $u$ satisfies the modified Camassa-Holm Equation (23) with $a=1$, where $m=k=u-u_{s s}$ is the curvature of the curve $\gamma$. Denote $\Lambda=1-\partial_{s}^{2}$, then $u=\Lambda^{-1} k$. Assume that $\tilde{\gamma}$ is another curve related to $\gamma$ by Equation (13), a direct computation shows

$$
\tilde{\gamma}_{t}=\left(u_{s}+\alpha_{t}+\beta\left(f_{s}+k h\right)\right) \mathbf{n}+\left(\frac{1}{2}\left(u^{2}-u_{s}^{2}\right)+\beta_{t}-\alpha\left(f_{s}+k h\right)\right) \mathbf{t} .
$$

Using Equation (19), the corresponding geometric invariants of $\tilde{\gamma}$ can be expressed in

$$
\begin{aligned}
\tilde{\Lambda} & =1-\partial_{\tilde{s}}^{2}=1-\left(\frac{d s}{d \tilde{s}} \partial_{s}\right)\left(\frac{d s}{d \tilde{s}} \partial_{s}\right), \\
\tilde{u} & =\left(1-\partial_{\tilde{s}}^{2}\right)^{-1} \tilde{k}, \\
\tilde{u}_{\tilde{s}} & \left.=1-\partial_{\tilde{s}}^{2}\right)^{-1} \tilde{k}_{\tilde{s}}, \\
\tilde{u}^{2}-\tilde{u}_{\tilde{s}}^{2} & =\left[\left(1-\partial_{\tilde{s}}^{2}\right)^{-1}\left(\tilde{k}+\tilde{k}_{\tilde{s}}\right)\right]\left[\left(1-\partial_{\tilde{s}}^{2}\right)^{-1}\left(\tilde{k}-\tilde{k}_{\tilde{s}}\right)\right],
\end{aligned}
$$


where $\tilde{k}=\sqrt{F_{1}^{2}+G_{1}^{2}} /\left(F_{0}^{2}+G_{0}^{2}\right)^{2}$. Assume that $\tilde{\gamma}$ is also governed by the modified Camassa-Holm flow (24), namely, it satisfies

$$
\tilde{\gamma}_{t}=\tilde{u}_{\tilde{s}} \tilde{\mathbf{n}}+\frac{1}{2}\left(\tilde{u}^{2}-\tilde{u}_{\tilde{s}}^{2}\right) \tilde{\mathbf{t}}
$$

Substituting Equations (15), (25) and (26) into Equation (27) and comparing the coefficients of $\mathbf{t}$ and $\mathbf{n}$ in the resulting equation, we arrive at the following system for $\alpha(t, s)$ and $\beta(t, s)$

$$
\begin{aligned}
& \alpha_{t}+u_{s}+\beta\left[u_{s s}+\frac{1}{2}\left(u-u_{s s}\right)\left(u^{2}-u_{s}^{2}\right)\right]=\frac{\tilde{u}_{\tilde{s}} F_{0}}{\sqrt{F_{0}^{2}+G_{0}^{2}}}+\frac{1}{2} \frac{\left(\tilde{u}^{2}-\tilde{u}_{\tilde{s}}^{2}\right)}{\sqrt{F_{0}^{2}+G_{0}^{2}}}, \\
& \beta_{t}+\frac{1}{2}\left(u^{2}-u_{s}^{2}\right)-\alpha\left[u_{s s}+\frac{1}{2}\left(u-u_{s s}\right)\left(u^{2}-u_{s}^{2}\right)\right]=-\frac{\tilde{u}_{\tilde{s}} G_{0}}{\sqrt{F_{0}^{2}+G_{0}^{2}}}+\frac{1}{2} \frac{\left(\tilde{u}^{2}-\tilde{u}_{\tilde{s}}^{2}\right)}{\sqrt{F_{0}^{2}+F_{0}^{2}}},
\end{aligned}
$$

where $u$ satisfies the modified Camassa-Holm Equation (23) with $a=1$. Consequently, we have the following result.

Theorem 2.2. The modified Camassa-Holm flow (24) is invariant with respect to the Bäcklund transformation (13) if $\alpha(t, s)$ and $\beta(t, s)$ satisfy the system (28), where $G_{0}$ and $F_{0}$ are given in Equation (16).

\section{Bäcklund Transformations for Space Curve Flows in $\mathbb{R}^{3}$}

In this section, we consider the integrable flows for space curves in $\mathbb{R}^{3}$

$$
\gamma_{t}=U \mathbf{n}+V \mathbf{b}+W \mathbf{t}
$$

where $\mathbf{t}, \mathbf{n}$ and $\mathbf{b}$ are the tangent, normal and binormal vectors of the space curve $\gamma$, respectively. The velocities $U, V$ and $W$ depend on the curvature and torsion as well as their derivatives with respect to arclength $s$. It is well know that the vectors $\mathbf{t}, \mathbf{n}$ and $\mathbf{b}$ satisfy the Serret-Frenet formulae

$$
\begin{aligned}
\mathbf{t}_{s} & =k \mathbf{n}, \\
\mathbf{n}_{s} & =-k \mathbf{t}+\tau \mathbf{b}, \\
\mathbf{b}_{s} & =-\tau \mathbf{n},
\end{aligned}
$$

where $k$ and $\tau$ are curvature and torsion of $\gamma$. Governed by the flow (29), the time evolutions of these geometric invariants fulfill $[14,15]$

$$
\begin{aligned}
\mathbf{t}_{t} & =\left(\frac{\partial U}{\partial s}-\tau V+k W\right) \mathbf{n}+\left(\frac{\partial V}{\partial s}+\tau U\right) \mathbf{b}, \\
\mathbf{n}_{t} & =-\left(\frac{\partial U}{\partial s}-\tau V+k W\right) \mathbf{t}+\left[\frac{1}{k} \frac{\partial}{\partial s}\left(\frac{\partial V}{\partial s}+\tau U\right)+\frac{\tau}{k}\left(\frac{\partial U}{\partial s}-\tau V+k W\right)\right] \mathbf{b}, \\
\mathbf{b}_{t} & =-\left(\frac{\partial V}{\partial s}+\tau U\right) \mathbf{t}-\left[\frac{1}{k} \frac{\partial}{\partial s}\left(\frac{\partial V}{\partial s}+\tau U\right)+\frac{\tau}{k}\left(\frac{\partial U}{\partial s}-\tau V+k W\right)\right] \mathbf{n}, \\
g_{t} & =g\left(\frac{\partial W}{\partial s}-k U\right),
\end{aligned}
$$

where $g=\left|\gamma_{p}\right|$ denotes the metric of the curve $\gamma$. A direct computation leads to the equations for the curvature $k$ and torsion $\tau$ :

$$
\begin{aligned}
& \frac{\partial \tau}{\partial t}=\frac{\partial}{\partial s}\left[\frac{1}{k} \frac{\partial}{\partial s}\left(\frac{\partial V}{\partial s}+\tau U\right)+\frac{\tau}{k}\left(\frac{\partial U}{\partial s}-\tau V\right)+\tau \int^{s} k U d s^{\prime}\right]+k \tau U+k \frac{\partial V}{\partial s}, \\
& \frac{\partial k}{\partial t}=\frac{\partial^{2} U}{\partial s^{2}}+\left(k^{2}-\tau^{2}\right) U+\frac{\partial k}{\partial s} \int^{s} k U d s^{\prime}-2 \tau \frac{\partial V}{\partial s}-k \frac{\partial \tau}{\partial s} V .
\end{aligned}
$$


Assume that the flow is intrinsic, namely the arclength does not depend on time, it implies from Equation (31) that

$$
\frac{\partial W}{\partial s}=k U
$$

From Equation (32), using the following Hasimoto transformation

$$
\phi=k \eta, \quad \eta=\exp \left[i \int^{s} \tau\left(t, s^{\prime}\right) d s^{\prime}\right]
$$

we get the equation for $\phi$

$$
\begin{aligned}
\frac{\partial \phi}{\partial t}= & \left(\frac{\partial^{2}}{\partial s^{2}}+|\phi|^{2}+i \phi \int^{s} d s^{\prime} \tau \phi^{*}+\frac{\partial \phi}{\partial s} \int^{s} d s^{\prime} \phi^{*}\right)(U \eta) \\
& +\left(i \frac{\partial^{2}}{\partial s^{2}}+i|\phi|^{2}+\phi \int^{s} d s^{\prime} \tau \phi^{*}-i \phi \int^{s} d s^{\prime} \frac{\partial \phi^{*}}{\partial s^{\prime}}\right)(V \eta)
\end{aligned}
$$

where $\phi^{*}$ denotes the complex conjugate of $\phi$.

Let $U=0, V=k$ and $W=0$. Then we derive from Equation (32) the Schrödinger equation

$$
i \phi_{t}+\phi_{s s}+\frac{1}{2}|\phi|^{2} \phi=0 \text {. }
$$

Let $U=-k_{s}, V=-k \tau$. Then $W=-\frac{1}{2} k^{2}$, and $\phi$ satisfies the mKdV system [15]

$$
\phi_{t}+\phi_{s s s}+\frac{3}{2}|\phi|^{2} \phi_{s}=0
$$

We now consider the case of $U=W=0$. Denote $\theta(t, s)=\int^{s} \tau\left(s^{\prime}, t\right) d s^{\prime}, G=V \eta$. It follows from Equation (35) that $\phi$ satisfies the equation

$$
i \phi_{t}+G_{s s}+|\phi|^{2} G-\phi \int^{s} G(\cos \theta-i \sin \theta) k_{s^{\prime}} d s^{\prime}=0
$$

Let $\tilde{u}=k \cos \theta, \tilde{v}=k \sin \theta$ and $G=G_{1}+i G_{2}$. Then Equation (33) is separated into the two equations

$$
\begin{aligned}
& \tilde{u}_{t}=-G_{2, s s}-\tilde{v} \partial_{s}^{-1}\left[k\left(G_{1} \cos \theta+G_{2} \sin \theta\right)_{s}\right], \\
& \tilde{v}_{t}=G_{1, s s}+\tilde{u} \partial_{s}^{-1}\left[k\left(G_{1} \cos \theta+G_{2} \sin \theta\right)_{s}\right] .
\end{aligned}
$$

Furthermore, letting $\tilde{u}=u+v_{s}, \tilde{v}=v-u_{s}$ and choosing $V=\partial_{s}^{-1}\left[\left(u^{2}+v_{s}^{2}\right) / k\right]$, we find that $u$ and $v$ satisfy the following system [41]

$$
\begin{aligned}
& \left(u+v_{s}\right)_{t}=-G_{2, s s}-\left(v-u_{s}\right)\left(u^{2}+v^{2}\right), \\
& \left(v-u_{s}\right)_{t}=G_{1, s s}+\left(u+v_{s}\right)\left(u^{2}+v^{2}\right),
\end{aligned}
$$

where $G_{1}=2 \cos \theta \partial_{s}^{-1}(v \cos \theta-u \sin \theta), G_{2}=2 \sin \theta \partial_{s}^{-1}(v \cos \theta-u \sin \theta)$, which is related to the dual system of the Schrödinger equation [42]. 


\subsection{The Schrödinger Flow}

Corresponding to the Schrödinger Equation (36), the Schrödinger flow is given by [14]

$$
\gamma_{t}=k \mathbf{b}
$$

In this case, the time evolution of frame vectors is governed by

$$
\begin{aligned}
\mathbf{t}_{t} & =-\tau k \mathbf{n}+k_{s} \mathbf{b}, \\
\mathbf{n}_{t} & =-\left(\frac{k_{s s}}{k}-\tau^{2}\right) \mathbf{b}+\tau k \mathbf{t} \\
\mathbf{b}_{t} & =-k_{s} \mathbf{t}-\left(\frac{k_{s s}}{k}-\tau^{2}\right) \mathbf{n} .
\end{aligned}
$$

We now construct Bäcklund transformation of the Schrödinger flow (40)

$$
\tilde{\gamma}=\gamma+\alpha(t, s) \mathbf{t}+\beta(t, s) \mathbf{n}+\chi(t, s) \mathbf{b},
$$

where $\alpha, \beta$ and $\chi$ are the functions of $t$ and $s$, to be determined. Using Equation (30), (40) and (41), a direct computation leads to

$$
\tilde{\gamma}_{s}=\left(1+\alpha_{s}-\beta k\right) \mathbf{t}+\left(\beta_{s}+\alpha k-\chi \tau\right) \mathbf{n}+\left(\chi_{s}+\beta \tau\right) \mathbf{b}
$$

and

$$
\begin{aligned}
\tilde{\gamma}_{t}=( & \left.\alpha_{t}+\beta \tau k-\chi k_{s}\right) \mathbf{t}+\left[\beta_{t}-\chi\left(\frac{k_{s s}}{k}-\tau^{2}\right)-\alpha \tau k\right] \mathbf{n} \\
+ & {\left[\chi_{t}+k+\alpha k_{s}+\beta\left(\frac{k_{s s}}{k}-\tau^{2}\right)\right] \mathbf{b} . }
\end{aligned}
$$

Then the arclength parameter $\tilde{s}$ of curve $\tilde{\gamma}$ is related to $s$ by

$$
d \tilde{s}=\left|\tilde{\gamma}_{s}\right| d s=\sqrt{\left(1+\alpha_{s}-\beta k\right)^{2}+\left(\beta_{s}+\alpha k-\chi \tau\right)^{2}+\left(\chi_{s}+\beta \tau\right)^{2}} d s:=F d s .
$$

The tangent vector of the curve $\tilde{\gamma}$ is determined by

$$
\tilde{\mathbf{t}}=\tilde{\gamma}_{s} \frac{d s}{d \tilde{s}}=A_{1} \mathbf{t}+A_{2} \mathbf{n}+A_{3} \mathbf{b}
$$

where $A_{1}=F^{-1}\left(1+\alpha_{s}-\beta k\right), A_{2}=F^{-1}\left(\beta_{s}+\alpha k-\chi \tau\right), A_{3}=F^{-1}\left(\chi_{s}+\beta \tau\right)$. Further computation from Equation (43) yields

$$
\tilde{\gamma}_{\tilde{s} \tilde{s}}=\tilde{\gamma}_{\tilde{s} s} \frac{d s}{d \tilde{s}}=\frac{A_{1 s}-k A_{2}}{F} \mathbf{t}+\frac{A_{2 s}+k A_{1}-\tau A_{3}}{F} \mathbf{n}+\frac{A_{3 s}+\tau A_{2}}{F} \mathbf{b},
$$

which gives the curvature of $\tilde{\gamma}$ :

$$
\tilde{k}=\frac{\sqrt{\left(A_{1 s}-k A_{2}\right)^{2}+\left(A_{2 s}+k A_{1}-\tau A_{3}\right)^{2}+\left(A_{3 s}+\tau A_{2}\right)^{2}}}{F}:=\frac{H}{F} .
$$

Using the Serret-Frenet formulae, we obtain the normal and binormal vectors of $\tilde{\gamma}$ given by

$$
\begin{aligned}
\tilde{\mathbf{n}} & =\frac{A_{1 s}-k A_{2}}{H} \mathbf{t}+\frac{A_{2 s}+k A_{1}-\tau A_{3}}{H} \mathbf{n}+\frac{A_{3 s}+\tau A_{2}}{H} \mathbf{b}:=B_{1} \mathbf{t}+B_{2} \mathbf{n}+B_{3} \mathbf{b}, \\
\tilde{\mathbf{b}} & =\frac{C_{1} \mathbf{t}+C_{2} \mathbf{n}+C_{3} \mathbf{b}}{\sqrt{C_{1}^{2}+C_{2}^{2}+C_{3}^{2}}},
\end{aligned}
$$


where $C_{1}=F^{-1}\left(B_{1 s}-k B_{2}+H A_{1}\right), C_{2}=F^{-1}\left(k B_{1}+B_{2 s}-\tau B_{3}+H A_{2}\right)$ and $C_{3}=F^{-1}\left(\tau B_{2}+\right.$ $\left.B_{3 s}+H_{3}\right)$.

Assume that the curve $\tilde{\gamma}$ also fulfills the Schrödinger flow, that is

$$
\tilde{\gamma}_{t}=\tilde{k} \tilde{\mathbf{b}}
$$

Plugging Equations (44), (45) and (46) into Equation (47), we arrive at the following result.

Theorem 3.1. The Schrödinger flow (40) is invariant with respect to the Bäcklund transformation (42) if $\alpha, \beta$ and $\chi$ satisfy the system

$$
\begin{aligned}
& \alpha_{t}+\beta \tau k-\chi k_{s}=\frac{H}{F} \frac{C_{1}}{\sqrt{C_{1}^{2}+C_{2}^{2}+C_{3}^{2}}}, \\
& \beta_{t}-\chi\left(\frac{k_{s s}}{k}-\tau^{2}\right)-\alpha \tau k=\frac{H}{F} \frac{C_{2}}{\sqrt{C_{1}^{2}+C_{2}^{2}+C_{3}^{2}}}, \\
& \chi_{t}+k+\alpha k_{s}+\beta\left(\frac{k_{s s}}{k}-\tau^{2}\right)=\frac{H}{F} \frac{C_{3}}{\sqrt{C_{1}^{2}+C_{2}^{2}+C_{3}^{2}}} .
\end{aligned}
$$

\subsection{The Extended Harry-Dym Flow}

The extended Harry-Dym flow [19]

$$
\gamma_{t}=\tau^{-\frac{1}{2}} \mathbf{b}
$$

is obtained by setting $U=M=0$, and $V=\tau^{-\frac{1}{2}}$ in the space curve flow (29). Here we consider the curve flow with constant curvature $k$. Let $k=1$, it follows from Equation (32) that the torsion of $\gamma$ satisfies the extended Harry-Dym equation [19]

$$
\tau_{t}=\left[\left(\tau^{-\frac{1}{2}}\right)_{s s}-\tau^{\frac{3}{2}}+\tau^{-\frac{1}{2}}\right]_{s},
$$

which is equivalent to the flow (48). Making use of the transformation $v=\tau^{-1 / 2}$, we get the equation

$$
\left(v^{-1}\right)_{t}=\frac{1}{2}\left(v v_{s s}-\frac{1}{2} v_{s}^{2}+\frac{1}{2} v^{2}-\frac{3}{2} v^{-2}\right)_{s} .
$$

In terms of the change of variables $d x=\sqrt{2} v^{-1} d s+\frac{1}{\sqrt{2}}\left(v v_{s s}-\frac{1}{2} v_{s}^{2}+\frac{1}{2} v^{2}-\frac{3}{2} v^{-2}\right) d t$, it is deduced that

$$
\frac{\partial v}{\partial t}=\frac{\partial}{\partial x}\left[v\left(\frac{\partial}{\partial x}\left(\frac{v_{x}}{v}\right)-\frac{1}{2}\left(\frac{v_{x}}{v}\right)^{2}\right)+\frac{1}{4} v^{3}-\frac{3}{4} v^{-1}\right] .
$$

Again we set $v=e^{\varphi}$, then it is inferred from Equation (50) that $\varphi$ satisfies the Calogero's modified $\mathrm{KdV}$ equation

$$
\varphi_{t}=\varphi_{x x x}-\frac{1}{2} \varphi_{x}^{3}+\frac{3}{2} \varphi_{x} \cosh 2 \varphi
$$

We now construct Bäcklund transformations to the extended Harry-Dym flow (48). In this case, the corresponding time evolution of frame vectors $\mathbf{t}, \mathbf{n}$ and $\mathbf{b}$ are given by

$$
\begin{aligned}
\mathbf{t}_{t} & =-\tau^{\frac{1}{2}} \mathbf{n}-\frac{1}{2} \tau^{-\frac{3}{2}} \tau_{s} \mathbf{b} \\
\mathbf{n}_{t} & =\tau^{\frac{1}{2}} \mathbf{t}+\left(\left(\tau^{-\frac{1}{2}}\right) s s-\tau^{\frac{3}{2}}\right) \mathbf{b} \\
\mathbf{b}_{t} & =\frac{1}{2} \tau^{-\frac{3}{2}} \tau_{s} \mathbf{t}-\left(\left(\tau^{-\frac{1}{2}}\right)_{s s}-\tau^{\frac{3}{2}}\right) \mathbf{n} .
\end{aligned}
$$


In terms of Equation (51), a direct computation gives

$$
\begin{aligned}
\tilde{\gamma}_{t}=[ & \left.\tau^{-\frac{1}{2}}+\alpha\left(\tau^{-\frac{1}{2}}\right)_{s}+\beta\left(\left(\tau^{-\frac{1}{2}}\right)_{s s}-\tau^{\frac{3}{2}}\right)+\chi_{t}\right] \mathbf{b}, \\
& +\left[\beta_{t}-\alpha \tau^{\frac{1}{2}}-\chi\left(\left(\tau^{-\frac{1}{2}}\right)_{s s}-\tau^{\frac{3}{2}}\right)\right] \mathbf{n}+\left[\alpha_{t}+\beta \tau^{\frac{1}{2}}+\chi\left(\tau^{-\frac{1}{2}}\right)_{s}\right] \mathbf{t} .
\end{aligned}
$$

Assume that a new curve $\tilde{\gamma}(t, s)$ is governed by the extended Harry-Dym flow, that means $\tilde{\gamma}$ satisfies

$$
\tilde{\gamma}_{t}=\tilde{\tau}^{-\frac{1}{2}} \tilde{\mathbf{b}}
$$

where $\tilde{\tau}$ and $\tilde{b}$ are the torsion and binormal vector of $\tilde{\gamma}$, respectively, which is related to the geometric invariants of $\gamma$ through

$$
\begin{aligned}
& \tilde{\tau}=\frac{C_{1}\left(B_{1, s}-k B_{2}\right)+C_{2}\left(B_{1} k+B_{2, s}-\tau B_{3}+H A_{2}\right)+C_{3}\left(\tau B_{2}+B_{3 s}+H A_{3}\right)}{F \sqrt{C_{1}^{2}+C_{2}^{2}+C_{3}^{2}}}, \\
& \tilde{\mathbf{b}}=\frac{C_{1} \mathbf{t}+C_{2} \mathbf{n}+C_{3} \mathbf{b}}{\sqrt{C_{1}^{2}+C_{2}^{2}+C_{3}^{2}}} .
\end{aligned}
$$

Plugging Equations (52) and (54) into Equation (53) implies that the extended Harry-Dym equation is invariant with respect to the Bäcklund transformation (42) if $\alpha, \beta$ and $\chi$ satisfy the following system

$$
\begin{aligned}
& \alpha_{t}+\beta \tau^{\frac{1}{2}}+\chi\left(\tau^{-\frac{1}{2}}\right)_{s}=\tilde{\tau}^{-\frac{1}{2}} \frac{C_{1}}{\sqrt{C_{1}^{2}+C_{2}^{2}+C_{3}^{2}}}, \\
& \beta_{t}-\alpha \tau^{\frac{1}{2}}-\chi\left(\left(\tau^{-\frac{1}{2}}\right)_{s s}-\tau^{\frac{3}{2}}\right)=\tilde{\tau}^{-\frac{1}{2}} \frac{C_{2}}{\sqrt{C_{1}^{2}+C_{2}^{2}+C_{3}^{2}}}, \\
& \chi_{t}+\tau^{-\frac{1}{2}}+\alpha\left(\tau^{-\frac{1}{2}}\right)_{s}+\beta\left(\left(\tau^{-\frac{1}{2}}\right)_{s s}-\tau^{\frac{3}{2}}\right)=\tilde{\tau}^{-\frac{1}{2}} \frac{C_{3}}{\sqrt{C_{1}^{2}+C_{2}^{2}+C_{3}^{2}}} .
\end{aligned}
$$

\section{Bäcklund Transformations of the KdV and Camassa-Holm Flows}

Integrable curve flows in the centro-equiaffine geometry were discussed extensively in $[21,24,33,35,40]$. It turns out that the $\mathrm{KdV}$ equation arises naturally from a non-stretching curve flow in centro-equiaffine geometry.

For a planar curve $\gamma(p)$ in the centro-equiaffine geometry, which satisfies $\left[\gamma, \gamma_{p}\right] \neq 0$, one can reparametrize it by the special parameter $s$ satisfying $\left[\gamma, \gamma_{s}\right]=1$, where the parameter $s$ is said to be centro-equiaffine arclength. It follows that in terms of the free parameter $p$, the centro-equiaffine arclength is represented by

$$
d s=\left[\gamma, \gamma_{p}\right] d p
$$

Furthermore, the centro-equiaffine curvature of the curve $\gamma(s)$ is defined to be

$$
\phi=\left[\gamma_{s}, \gamma_{s s}\right]
$$

Consider the planar curve flow in the centro-equiaffine geometry, specified by

$$
\gamma_{t}=f \mathbf{N}+h \mathbf{T}
$$

where $\mathbf{N}$ and $\mathbf{T}$ are normal and tangent vectors of $\gamma$. One can compute the time evolution of $\mathbf{N}$ and $\mathbf{T}$ to get

$$
\left(\begin{array}{c}
\mathbf{T} \\
\mathbf{N}
\end{array}\right)_{t}=\left(\begin{array}{cc}
h_{s}-f & f_{s}+\phi h \\
-h & -f
\end{array}\right)\left(\begin{array}{c}
\mathbf{T} \\
\mathbf{N}
\end{array}\right)
$$


The Serret-Frenet formulae for curves in centro-equiaffine geometry reads

$$
\mathbf{T}_{s}=\phi \mathbf{N}, \quad \mathbf{N}_{s}=-\mathbf{T}
$$

Assume that the flow is intrinsic, a direct computation shows that the curvature $\phi$ satisfies

$$
\phi_{t}=\left(D_{s}^{2}+4 \phi+2 \phi_{s} \partial^{-1}\right) f .
$$

Letting $f=\phi_{s}$ in Equation (58), we get the $\mathrm{KdV}$ equation

$$
\phi_{t}=\phi_{s s s}+6 \phi \phi_{s}
$$

The corresponding KdV flow is

$$
\gamma_{t}=\phi_{s} \mathbf{N}+2 \phi \mathbf{T}
$$

which was introduced firstly by Pinkall [18]. Now we consider the Bäcklund transformation of the KdV flow (60)

$$
\tilde{\gamma}(t, s)=\gamma(t, s)+\alpha \mathbf{N}+\beta \mathbf{T}
$$

where $\alpha$ and $\beta$ are functions of $t$ and $s$.

We now construct the Bäcklund transformations of the KdV flow. Differentiating Equation (61) with respect to $t$ and using Equation (60), we get

$$
\tilde{\gamma}_{t}=\left[\alpha_{t}+(1-\alpha) \phi_{s}+\beta\left(\phi_{s s}+2 \phi^{2}\right)\right] \mathbf{N}+\left[2(1-\alpha) \phi+\beta_{t}+\beta \phi_{s}\right] \mathbf{T} .
$$

Assume that the curve $\tilde{\gamma}$ is also governed by the KdV flow, namely it satisfies

$$
\tilde{\gamma}_{t}=\tilde{\phi}_{\tilde{s}} \tilde{\mathbf{N}}+2 \tilde{\phi} \tilde{\mathbf{T}}
$$

where $\tilde{s}$ is the arclength of $\tilde{\gamma}$, which satisfies $d \tilde{s}=\left(1-\alpha+\beta_{s}\right) d s$. In Equation (63), $\tilde{\mathbf{T}}$ and $\tilde{\mathbf{N}}$ are tangent and normal vectors of $\tilde{\gamma}$, which are related to $\mathbf{T}$ and $\mathbf{N}$ through

$$
\begin{aligned}
& \tilde{\mathbf{T}}=\tilde{\gamma}_{\tilde{s}}=\tilde{\gamma}_{s} \frac{d s}{d \tilde{s}}=\mathbf{T}+\frac{\alpha_{s}+\beta \phi}{1-\alpha+\beta_{s}} \mathbf{N}, \\
& \tilde{\mathbf{N}}=-\tilde{\gamma}=-\beta \mathbf{T}+(1-\alpha) \mathbf{N} .
\end{aligned}
$$

Further computation using Equation (62) leads to

$$
\tilde{\gamma}_{\tilde{s} \tilde{s}}=\tilde{\gamma}_{\tilde{s} s} \frac{d s}{d \tilde{s}}=\frac{\phi+\left(\frac{\alpha_{s}+\beta \phi}{1-\alpha+\beta_{s}}\right)_{s}}{1-\alpha+\beta_{s}} \mathbf{N}-\frac{\alpha_{s}+\beta \phi}{\left(1-\alpha+\beta_{s}\right)^{2}} \mathbf{T} .
$$

It follows from Equations (64) and (65) that the centro-equiaffine curvature of $\tilde{\gamma}$ is given by

$$
\tilde{\phi}=\left[\tilde{\gamma}_{\tilde{s}}, \tilde{\gamma}_{\tilde{s} \tilde{s}}\right]=\frac{\phi+\left(\frac{\alpha_{s}+\beta \phi}{1-\alpha+\beta_{s}}\right)_{s}}{1-\alpha+\beta_{s}}+\frac{\alpha_{s}+\beta \phi}{\left(1-\alpha+\beta_{s}\right)^{3}} .
$$

Plugging Equations (64) and (66) into the right hand side of Equation (63), and comparing the coefficients of $\mathbf{T}$ and $\mathbf{N}$ with Equation (62), we deduce the following result. 
Theorem 4.1. The KdV flow is invariant with respect to the Bäcklund transformation (61) if $\alpha$ and $\beta$ satisfy the system

$$
\begin{aligned}
& \alpha_{t}+(1-\alpha) \phi_{s}+\beta\left(\phi_{s s}+2 \phi^{2}\right)=(1-\alpha) \tilde{\phi}_{\tilde{s}}+2 \frac{\alpha_{s}+\beta \phi}{1-\alpha+\beta_{s}} \tilde{\phi}, \\
& \beta_{t}-2(1-\alpha) \phi+\beta \phi_{s}=2 \tilde{\phi}-\beta \tilde{\phi}_{\tilde{s}},
\end{aligned}
$$

where $\tilde{\phi}$ is determined by Equation (66).

Example 4.1. It is easy to see that $\phi=0$ is a trivial solution of the $\mathrm{KdV}$ equation. Let $\phi=0$, then

$$
\tilde{\phi}=\frac{\left(\frac{\alpha_{s}}{1-\alpha+\beta_{s}}\right)_{s}}{1-\alpha+\beta_{s}}+\frac{\alpha_{s}}{\left(1-\alpha+\beta_{s}\right)^{3}},
$$

and system (67) becomes

$$
\left\{\begin{array}{l}
\alpha_{t}=(1-\alpha)\left(\alpha_{\tilde{s} \tilde{s}}+\frac{\alpha_{\tilde{s}}}{\left(1-\alpha+\beta_{s}\right)^{2}}\right)_{\tilde{s}}+2 \alpha_{\tilde{s}}\left(\alpha_{\tilde{s} \tilde{s}}+\frac{\alpha_{\tilde{s}}}{\left(1-\alpha+\beta_{s}\right)^{2}}\right), \\
\beta_{t}=2\left(\alpha_{\tilde{s} \tilde{s}}+\frac{\alpha_{\tilde{s}}}{\left(1-\alpha+\beta_{s}\right)^{2}}\right)-\beta\left(\alpha_{\tilde{s} \tilde{s}}+\frac{\alpha_{\tilde{s}}}{\left(1-\alpha+\beta_{s}\right)^{2}}\right)_{\tilde{s}} .
\end{array}\right.
$$

This is a third-order quasi-linear system, it is difficult to solve it. For simplicity, we seek its time-independent solutions: $\alpha=\alpha(\tilde{s}), \beta=\beta(\tilde{s})$. Denote

$$
H=\alpha_{\tilde{s} \tilde{s}}+\frac{\alpha_{\tilde{s}}}{\left(1-\alpha+\beta_{s}\right)^{2}} .
$$

Then system (68) reduces to

$$
\begin{gathered}
(1-\alpha) H_{\tilde{s}}+2 \alpha_{\tilde{s}} H=0, \\
\beta H_{\tilde{s}}-2 H=0 .
\end{gathered}
$$

Integrating it, we arrive at

$$
H=c_{0}(1-\alpha)^{2}, \quad \beta=\frac{\alpha-1}{\alpha_{\tilde{s}}}
$$

where $c_{0} \neq 0$ is an integration constant. Employing the chain rule and $d \tilde{s}=\left(1-\alpha+\beta_{s}\right) d s$, we have

$$
\beta_{s}=\left(1-\alpha+\beta_{s}\right) \beta_{\tilde{s}}
$$

Solving it for $\beta_{s}$, we obtain

$$
\beta_{s}=\frac{(1-\alpha) \beta_{\tilde{s}}}{1-\beta_{\tilde{s}}} .
$$

A direct computation using Equation (69) yields

$$
\beta_{\tilde{s}}=1+\frac{(1-\alpha) \alpha_{\tilde{s} \tilde{s}}}{\alpha_{\tilde{s}}^{2}} .
$$

It follows from the above two equations that

$$
1-\alpha+\beta_{s}=\frac{\alpha_{\tilde{s}}^{2}}{\alpha_{\tilde{s} \tilde{s}}}
$$


In terms of $\alpha_{\tilde{s}}, H$ can be denoted as

$$
H=\alpha_{\tilde{s} \tilde{s}}+\frac{\alpha_{\tilde{s} \tilde{s}}^{2}}{\alpha_{\tilde{s}}^{3}}
$$

Hence the first equation in Equation (69) becomes

$$
\alpha_{\tilde{s} \tilde{s}}+\frac{\alpha_{\tilde{s} \tilde{s}}^{2}}{\alpha_{\tilde{s}}^{3}}-c_{0}(1-\alpha)^{2}=0 .
$$

Using the hodograph transformation

$$
y=1-\alpha(\tilde{s}), \quad \tilde{s}=w(y)
$$

we get the equation for $w(y)$

$$
w_{y}^{-3}\left(w_{y y}^{2}-w_{y y}\right)-c_{0} y^{2}=0 .
$$

This equation is reduced to the first-order ordinary differential equation

$$
h^{-3}\left(h_{y}^{2}-h_{y}\right)-c_{0} y^{2}=0
$$

by setting $h=w_{y}$. Consequently, we derive a Bäcklund transformation (61) of the KdV flow (60), where $\alpha(\tilde{s})$ satisfies Equation (70) and $\beta(\tilde{s})=\left(\alpha(\tilde{s}-1) / \alpha_{\tilde{s}}(\tilde{s})\right.$.

Next we consider the Bäcklund transformation of the Camassa-Holm flow. Let $f=v_{s}(t, s)$ and $g=2 v(t, s), v=\left(1-\partial_{s}^{2}\right)^{-1} \phi$, then flow (55) becomes

$$
\gamma_{t}=v_{s} \mathbf{N}+2 v \mathbf{T}
$$

which gives the Camassa-Holm equation $[6,44]$

$$
v_{t}-v_{s s t}+v_{s s s}+6 v v_{s}-4 v_{s} v_{s s}-2 v v_{s s s}=0
$$

Therefore, Equation (71) is called the Camassa-Holm flow. Similar to the discussion for the modified Camassa-Holm equation, we have the following result.

Theorem 4.2. The Camassa-Holm flow (71) admits the the Bäcklund transformation (61) if $\alpha(t, s)$ and $\beta(t, s)$ satisfy the system

$$
\begin{aligned}
& \alpha_{t}+(1-\alpha) v_{s}+\beta\left((1-2 v) v_{s s}+2 v^{2}\right)=(1-\alpha) \tilde{v}_{\tilde{s}}+2 \tilde{v} G_{1}, \\
& \beta_{t}+2 v(1-\alpha)+\beta v_{s}=2 \tilde{v} F_{1}-\beta \tilde{v}_{\tilde{s}},
\end{aligned}
$$

where $\tilde{s}$ is the arclength of $\tilde{\gamma}$, determined by $d \tilde{s}=\left[(1-\alpha)\left(1-\alpha+\beta_{s}\right)+\beta\left(\alpha_{s}+\phi \beta\right)\right] d s$, $\tilde{v}=\left(1-\partial_{\tilde{s}}^{2}\right)^{-1}\left(F_{1} G_{2}-G_{1} F_{2}\right)$, with

$$
\begin{aligned}
& F_{1}=\frac{1-\alpha+\beta_{s}}{H}, \quad G_{1}=\frac{\alpha_{s}+\beta \phi}{H}, \\
& F_{2}=\frac{F_{1, s}-G_{1}}{H}, \quad G_{2}=\frac{G_{1, s}+\phi F_{1}}{H}, \\
& H=(1-\alpha)\left(1-\alpha+\beta_{s}\right)+\beta\left(\alpha_{s}+\beta \phi\right) .
\end{aligned}
$$




\section{Bäcklund Transformations of the Sawada-Kotera Flow}

Motions of curves in the affine geometry were discussed in [13,21,23,33,40]. It is well-known that the Sawada-Kotera equation arises from a non-stretching curve flow in affine geometry.

For a planar curve $\gamma(p)$ satisfying $\left[\gamma_{p}, \gamma_{p p}\right] \neq 0$ in affine geometry, we can reparametrize it by the special parameter $s$ satisfying $\left[\gamma_{s}, \gamma_{s s}\right]=1$, where the parameter $s$ is said to be the arclength. So the affine arclength can be expressed by

$$
d s=\left[\gamma_{p}, \gamma_{p p}\right]^{\frac{1}{3}} d p
$$

Consider the planar curve flow in affine geometry, governed by

$$
\gamma_{t}=f \mathbf{N}+h \mathbf{T}
$$

where $\mathbf{N}$ and $\mathbf{T}$ are affine normal and tangent vectors of $\gamma$. The Serret-Frenet formulae for curves in affine geometry reads

$$
\mathbf{T}_{s}=\mathbf{N}, \quad \mathbf{N}_{s}=-\mu \mathbf{T},
$$

where $\mu$ is the curvature of the curve $\gamma$, defined by

$$
\mu=\left[\gamma_{s s}, \gamma_{s s s}\right]
$$

One can compute the time evolution of $\mathbf{N}$ and $\mathbf{T}$, to get

$$
\left(\begin{array}{c}
\mathbf{T} \\
\mathbf{N}
\end{array}\right)_{t}=\left(\begin{array}{cc}
h_{s}-\mu f & f_{s}+\mu h \\
H_{1} & H_{2}
\end{array}\right)\left(\begin{array}{c}
\mathbf{T} \\
\mathbf{N}
\end{array}\right)
$$

where $H_{1}=h_{s s}-2 \mu f_{s}-\mu h, H_{2}=f_{s s}+2 h_{s}-\mu f$. Assume that the flow is intrinsic, that means the arclength does not depend on time. It is inferred from $\left[\frac{\partial}{\partial t}, \frac{\partial}{\partial s}\right]=0$ that

$$
h=-\frac{1}{3} f_{s}+\frac{2}{3} \partial_{s}^{-1}(\mu f) .
$$

A direct computation gives the equation for the curvature [21]

$$
\mu_{t}=\frac{1}{3}\left(D_{s}^{4}+5 \mu D_{s}^{2}+4 \mu_{s} D_{s}+\mu_{s s}+4 \mu^{2}+2 \mu_{s} \partial^{-1} \mu\right) f .
$$

Letting $f=-3 \mu_{s}$ in Equation (77), we obtain the Sawada-Kotera equation [45]

$$
\mu_{t}+\mu_{5}+5 \mu \mu_{3}+5 \mu_{1} \mu_{2}+5 \mu^{2} \mu_{1}=0 \text {. }
$$

The corresponding Sawada-Kotera flow is [21]

$$
\gamma_{t}=-3 \mu_{s} \mathbf{N}+\left(\mu_{s s}-\mu^{2}\right) \mathbf{T}
$$

We now consider the Bäcklund transformation of the Sawada-Kotera flow (79), determined by Equation (61), where $\mathbf{N}$ and $\mathbf{T}$ are respectively the affine normal and tangent of $\gamma, \alpha(t, s)$ and $\beta(t, s)$ depend on $t$ and $s$. 
Using the Serret-Frenet formulae (74) and the Sawada-Kotera flow (79), we first have

$$
\begin{aligned}
\tilde{\gamma}_{t}= & {\left[\alpha_{t}-3 \mu_{s}-\left(\mu_{3}+\mu \mu_{s}\right) \alpha-\left(2 \mu_{s s}+\mu^{2}\right) \beta\right] \mathbf{N} } \\
& +\left[\beta_{t}+\mu_{s s}-\mu^{2}+\left(\mu_{4}+3 \mu \mu_{2}+\mu_{s}^{2}+\mu^{2}\right) \alpha+\left(\mu_{3}+\mu \mu_{s}\right) \beta\right] \mathbf{T} .
\end{aligned}
$$

On the other hand, assume that the new curve $\tilde{\gamma}$ is also governed by the Sawada-Kotera flow, which satisfies

$$
\tilde{\gamma}_{t}=-3 \tilde{\mu}_{\tilde{s}} \tilde{\mathbf{N}}+\left(\tilde{\mu}_{\tilde{s} \tilde{s}}-\tilde{\mu}^{2}\right) \tilde{\mathbf{T}}
$$

where $\tilde{s}$ is the arclength of $\tilde{\gamma}$, defined by $d \tilde{s}=\left[\tilde{\gamma}_{s}, \tilde{\gamma}_{s s}\right]^{\frac{1}{3}} d s$. In terms of the Sawada-Kotera flow, a direct computation yields

$$
\begin{aligned}
\tilde{\gamma}_{s} & =\left(1-\alpha \mu+\beta_{s}\right) \mathbf{T}+\left(\alpha_{s}+\beta\right) \mathbf{N}:=F_{1} \mathbf{T}+F_{2} \mathbf{N}, \\
\tilde{\gamma}_{s s} & =\left(F_{1, s}-\mu F_{2}\right) \mathbf{T}+\left(F_{1}+F_{2, s}\right) \mathbf{N}:=F_{3} \mathbf{T}+F_{4} \mathbf{N} .
\end{aligned}
$$

Thus the arclength parameter of $\tilde{\gamma}$ can be determined by

$$
d \tilde{s}=\left(F_{1}^{2}+F_{1} F_{2, s}-F_{2} F_{1, s}+\mu F_{2}^{2}\right)^{\frac{1}{3}} d s:=H_{1} d s .
$$

Using this and the flow (61), one can determine the tangent and normal vectors of $\tilde{\gamma}$ by

$$
\begin{aligned}
\tilde{\mathbf{T}}= & \frac{1}{H_{1}}\left(F_{1} \mathbf{T}+F_{2} \mathbf{N}\right), \\
\tilde{\mathbf{N}}= & \frac{1}{H_{1}}\left[\left(\left(\frac{F_{1}}{H_{1}}\right)_{s}-\mu \frac{F_{2}}{H_{1}}\right) \mathbf{T}+\left(\frac{F_{1}}{H_{1}}+\left(\frac{F_{2}}{H_{1}}\right)_{s}\right) \mathbf{N}\right] \\
& :=H_{2} \mathbf{T}+H_{3} \mathbf{N} .
\end{aligned}
$$

Thus the affine curvature of $\tilde{\gamma}$ is

$$
\tilde{\mu}=H_{2}\left(H_{2}+H_{3, s}\right)-H_{3}\left(H_{2, s}-\mu H_{3}\right):=H_{4} .
$$

Further computation gives

$$
\tilde{\mu}_{\tilde{s}}=\frac{H_{4, s}}{H_{1}}:=H_{5}, \quad \tilde{\mu}_{\tilde{s} \tilde{s}}=\frac{1}{H_{1}}\left(\frac{H_{4, s}}{H_{1}}\right)_{s}:=H_{6} .
$$

It follows that

$$
\begin{aligned}
& -3 \tilde{\mu}_{\tilde{s}} \tilde{\mathbf{N}}+\left(\tilde{\mu}_{\tilde{s} \tilde{s}}-\tilde{\mu}^{2}\right) \tilde{\mathbf{T}} \\
& \quad=\left[-3 H_{2} H_{5}+\frac{F_{1}}{H_{1}}\left(H_{6}-H_{4}^{2}\right)\right] \mathbf{T}+\left[-3 H_{3} H_{5}+\frac{F_{2}}{H_{1}}\left(H_{6}-H_{4}^{2}\right)\right] \mathbf{N} .
\end{aligned}
$$

Hence we have proved the following result.

Theorem 5.1. The Sawada-Kotera flow (79) is invariant with respect to the Bäcklund transformation (61) if $\alpha$ and $\beta$ satisfy the system

$$
\begin{aligned}
& \alpha_{t}-\left(\mu_{3}+\mu \mu_{s}\right) \alpha-3 \mu_{s}-\left(2 \mu_{s s}+\mu^{2}\right) \beta=-3 H_{3} H_{5}+\frac{F_{2}}{H_{1}}\left(H_{6}-H_{4}^{2}\right), \\
& \beta_{t}+\left(\mu_{3}+\mu \mu_{s}\right) \beta+\left(\mu_{4}+3 \mu \mu_{s s}+\mu_{s}^{2}+\mu^{2}\right) \alpha+\mu_{s s}-\mu^{2}=-3 H_{2} H_{5}+\frac{F_{1}}{H_{1}}\left(H_{6}-H_{4}^{2}\right) .
\end{aligned}
$$




\section{Acknowledgments}

The work of Changzheng Qu is supported by the NSF of China (Grant No. 11471174) and NSF of Ningbo (Grant No. 2014A610018). The work of Han is supported by Zhejiang Provincial NSF of China (Grant No. LQ12A01002) and Development Project for Visitors at Universities at Zhejiang Province (Grant No. FX2012013). The work of Kang is supported partially by the NSF of China (Grant No. 11471260).

\section{Author Contributions}

Changzheng Qu proposed the idea to study Bäcklund transformations of geometric curve flows. He discussed Bäcklund transformations of geometric curve flows in $\mathbb{R}^{2}$ and centro-equiaffine geometries, and provided detailed proofs to Theorem 2.1, 2.2, 4.1 and 4.2. Jingwei Han studied Bäcklund transformations of geometric curve flows in $\mathbb{R}^{3}$, and provided a detailed proof to Theorem 3.1, and carried out computation on the extended Harry-Dym flow. Jing Kang studied Bäcklund transformations of geometric curve flows in affine geometry, and provided a detailed proof to Theorem 5.1. Example 4.1 is given by Changzheng Qu and Jing Kang. Introduction is prepared by Changzheng Qu and Jingwei Han.

\section{Conflicts of Interest}

The authors declare no conflict of interest.

\section{References}

1. Bäcklund, A.V. Concerning Surfaces with Constant Negative Curvature; Coddington, E.M., Translator; New Era Printing Co.: Lancaster, PA, USA, 1905.

2. Rogers, C.; Schief, W.K. Bäcklund and Darboux Transformations Geometry and Modern Applications in Soliton Theory; Cambridge University Press: Cambridge, UK, 2002.

3. Chern, S.S.; Tenenblat, K. Pseudospherical surfaces and evolution equations. Stud. Appl. Math. 1986, 74, 55-83.

4. Wahlquist, H.D.; Estabrook, F.B. Prolongation structures of nonlinear evolution equations. J. Math. Phys. 1975, 16, 1-7.

5. Hirota, R. The Direct Method in Soliton Theory; Translated from the 1992 Japanese original and edited by Nagal, A.; Nimmo, J.; Gilson, C. With foreword by Hietarinta, J. and Nimmo, J.; Cambridge University Press: Cambridge, UK, 2004.

6. Fuchssteiner, B.; Fokas, A.S. Symplectic structures, their Bäcklund transformations and hereditary symmetries. Phys. D 1981/1982, 4, 47-66.

7. Chern, S.S.; Terng, C.L. An analogue of Bäcklund theorem in affine geometry. Rocky Montain Math. J. 1980, 10, 105-124.

8. Tenenblat, K.; Terng, C.L. Bäcklund theorem for $n$-dimensional submanifolds of $\mathbb{R}^{2 n-1}$. Ann. Math. 1980, 111, 477-490.

9. Terng, C.L.; Uhlenbeck, K. Bäcklund transformations and loop group actions. Commun. Pur. Appl. Math. 2000, 53, 1-75. 
10. Hirota, R.; Hu, X.B.; Tang, X.Y. A vector potential KdV equation and vector Ito equation: Soliton solutions, bilinear Bäcklund transformations and Lax-pairs. J. Math. Anal. Appl. 2003, 288, 326-348.

11. Calini, A.; Ivey, T. Bäcklund transformations and knots of constant torsion. J. Knot Theor. Ramf. 1998, 7, 719-746.

12. Inoguchi, J.; Kajiwara, K.; Matsuura, N.; Ohta, Y. Motion and Backlund transformations of discrete plane curves. Kyushu J. Math. 2012, 66, 303-324.

13. Olver, P.J. Equivalence, Invariants, and Symmetry; Cambridge University Press: Cambridge, UK, 1995.

14. Hasimoto, H. A soliton on a vortext filament. J. Fluid Mech. 1972, 51, 477-485.

15. Nakayama, K.; Segur, H.; Wadati, M. Integrability and the motion of curves. Phys. Rev. Lett. 1992, 16, 2603-2606.

16. Lamb, G.L. Soliton on moving space curves. J. Math. Phys. 1977, 18, 1654-1661.

17. Goldstein, R.E.; Petrich, D.M. The Korteweg-de Vries hierarchy as dynamics of closed curves in the plane. Phys. Rev. Lett. 1991, 67, 3203-3206.

18. Pinkall, U. Hamiltonian flows on the space of star-shaped curves. Result. Math. 1995, 27, 328-332.

19. Schief, W.K.; Rogers, C. Binormal motion of curves of constant curvature and torsion. Generation of soliton surfaces. R. Soc. Lond. Proc. Ser. A Math. Phys. Eng. Sci. 1999, 455, 3163-3188.

20. Ivey, T. Integrable geometric evolution equations for curves. Contemp. Math. 2001, 285, 71-84.

21. Chou, K.S.; Qu, C.Z. Integrable equations arising from motions of plane curves. Phys. D 2002, 162, 9-33.

22. Chou, K.S.; Qu, C.Z. Integrable equations arising from motions of plane curves II. J. Nonlinear Sci. 2003, 13, 487-517.

23. Chou, K.S.; Qu, C.Z. Integrable motions of space curves in affine geometry. Chaos Solitons Fractals 2002, 14, 29-44.

24. Chou, K.S.; Qu, C.Z. The KdV equation and motion of plane curves. J. Phys. Soc. Jan. 2001, 70, 1912-1916.

25. Marí Beffa, G.; Sanders, J.A.; Wang, J.P. Integrable systems in three-dimensional Riemannian geometry. J. Nonlinear Sci. 2002, 12, 143-167.

26. Calini, A.; Ivey, T. Finite-gap solutions of the vortex filament equation genus one solutions and symmetric solutions. J. Nonlinear Sci. 2005, 15, 321-361.

27. Marí Beffa, G. Hamiltonian evolution of curves in classical affine geometries. Phys. D 2009, 238, $100-115$.

28. Marí Beffa, G. Bi-Hamiltonian flows and their realizations as curves in real semisimple homogeneous manifolds. Pac. J. Math. 2010, 247, 163-188.

29. Marí Beffa, G.; Olver, P.J. Poisson structure for geometric curve flows in semi-simple homogeneous spaces. Regul. Chaotic. Dyn. 2010, 15, 532-550.

30. Anco, S.C. Bi-Hamitonian operators, integrable flows of curves using moving frames and geometric map equations. J. Phys. A Math. Gen. 2006, 39, 2043-2072.

31. Anco, S.C. Hamitonian flows of curves in $\mathrm{G} / \mathrm{SO}(\mathrm{N})$ and hyperbolic/evolutionary vector soliton equations. SIGMA 2006, 2, 1-17. 
32. Anco, S.C. Group-invariant solution equations and bi-Hamitonian geometric curve flows in Riemannian symplectic spaces. J. Geom. Phys. 2008, 58, 1-27.

33. Marí Beffa, G. Hamiltonian evolutions of curves in classical affine geometries. Phys. D 2009, 238, $100-115$.

34. Olver, P.J. Invariant submanifld flows. J. Phys. A 2008, 41, doi:10.1088/1751-8113/41/34/344017.

35. Calini, A.; Ivey, T.; Marí Beffa, G. Remarks on KdV-type flows on star-shaped curves. Phys. $D$ 2009, 238, 788-797.

36. Wo, W.F.; Qu, C.Z. Integrable motions of curves in $\mathbb{S}^{1} \times \mathbb{R}$. J. Geom. Phys. 2007, 57, 1733-1755.

37. Li, Y.Y.; Qu, C.Z.; Shu, S.C. Integrable motions of curves in projective geometries. J. Geom. Phys. 2010, 60, 972-985.

38. Song, J.F.; Qu, C.Z. Integrable systems and invariant curve flows in centro-equiaffine symplectic geometry. Phys. D 2012, 241, 393-402.

39. Musso, E. Motion of curves in the projective plane introducing the Kaup-Kuperschmidt hierarchy. SIGMA 2012, 8, doi:10.3842/SIGMA.2012.030.

40. Calini, A.; Ivey, T.; Marí Beffa, G. Integrable flows for starlike curves in centroaffine space. SIGMA 2013, 9, doi:10.3842/SIGMA.2013.022.

41. Qu, C.Z.; Song, J.F.; Yao, R.X. Multi-Component Intergrable Systems and Invariant Curve Flow in Certain Geometries. SIGMA 2013, 9, doi:10.3842/SIGMA.2013.001.

42. Olver, P.J.; Rosenau, P. Tri-Hamiltonian duality between solitons and solitary-wave solutions having compact support. Phys. Rev. E 1996, 53, 1900-1906.

43. Gui, G.L.; Liu, Y.; Olver, P.J.; Qu, C.Z. Wave-breaking and peakons for a modified Camassa-Holm equation. Commun. Math. Phys. 2013, 319, 731-759.

44. Camassa, R.; Holm, D.D. An integrable shallow water equation with peaked solitons. Phys. Rev. Lett. 1993, 71, 1661-1664.

45. Sawada, K.; Kotera, T. A method for finding N-soliton solutions of the KdV equation and KdV-like equation. Prog. Theor. Phys. 1974, 51, 1335-1367.

(c) 2015 by the authors; licensee MDPI, Basel, Switzerland. This article is an open access article distributed under the terms and conditions of the Creative Commons Attribution license (http://creativecommons.org/licenses/by/4.0/). 